(n)

\title{
Cross-sectional area of the median nerve is increased in primary autoimmune hypothyroidism and decreases upon treatment with thyroxine
}

\author{
Dana Holováčová, Martin Kužma, Zdenko Killinger and Juraj Payer \\ Comenius University Faculty of Medicine, 5th Department of Internal Medicine, University Hospital, \\ Bratislava, Slovakia
}

Correspondence should be addressed to D Holováčová Email

danaholovacova@gmail.com

\begin{abstract}
Background: The purpose of this study was to assess changes in the cross-sectional area (CSA) of nervus medianus by ultrasound in newly diagnosed primary hypothyroid patients after thyroxine replacement treatment.

Patients and methods: The cohort comprised 30 patients with newly diagnosed primary autoimmune hypothyroidism. These were subsequently compared with 30 controls, matched for sex, BMI and age. Ultrasound evaluation, including measurement of CSA at the level of the pisiform bone or tunnel inlet was performed at baseline and after 3 months. A CSA threshold of more than $10 \mathrm{~mm}^{2}$ was considered pathological.

Results: CSA was increased in patients compared with controls $\left(9.8 \pm 0.7 \mathrm{~mm}^{2}\right.$ vs $\left.7.2 \pm 1.1 \mathrm{~mm}^{2} ; P<0.001\right)$. Thyroxin substitution increased $\mathrm{fT}_{4}$ levels (baseline, $11.0 \pm 0.6 \mathrm{pmol} / \mathrm{L}$ vs $15.5 \pm 0.4 \mathrm{pmol} / \mathrm{L} ; P<0.001$ ) and decreased TSH (baseline, $23.9 \pm 6.0 \mathrm{mIU} / \mathrm{L}$ vs $2.7 \pm 0.2 \mathrm{mIU} / \mathrm{L} ; P<0.001)$. Thyroxine treatment decreased CSA to $8.4 \mathrm{~mm}^{2} \pm 0.3 \mathrm{~mm}^{2}(P=0.033)$.

Before treatment, four patients experienced distal paresthesia in the median nerve distribution area. Increased CSA pathognomonic for carpal tunnel syndrome found in two symptomatic patients normalized after 3 months. No clinical symptoms were observed after 3 months. A positive correlation was found between TSH and CSA $(R=0.155, P=0.05)$ before treatment.

Conclusion: Our study demonstrates that increase in median nerve CSA caused by primary autoimmune hypothyroidism can be fully reversible after achieving target levels of TSH and $\mathrm{fT}_{4}$.

\section{Introduction}

Thyroid hormones are involved in many functions of the central and peripheral nervous systems and, as a result, hypothyroidism may cause various neurological signs and symptoms (1). Chronic autoimmune thyroiditis is the most prevalent organ-specific autoimmune disease characterized by the presence of antibodies against thyroglobulin (anti-TG) and thyroid peroxidase (antiTPO) (2). Autoimmune diseases have a broad spectrum of clinical manifestations. Among them, neurologic involvement, from both the central and the peripheral nervous systems, is one of the most challenging
() 2016 European Society of Endocrinology Printed in Great Britain manifestations, regarding the diagnosis and treatment of autoimmune diseases (3). Several studies that have examined nerve conduction parameters in hypothyroid patients reported that deficiency of thyroid hormones causes motor neuropathy by affecting different peripheral nerves, but, more commonly, the median nerve $(4,5,6)$. A number of retrospective studies reported the prevalence of neuropathy to be between 10 and 70\% in patients with hypothyroidism $(1,7,8,9)$. The most common form of peripheral entrapment neuropathy is carpal tunnel syndrome (CTS) (10). According to the

Published by Bioscientifica Ltd. 
American Academy of Orthopedic Surgeons (AAOS), CTS is defined as a symptomatic median nerve compression neuropathy at the level of the wrist (11). The pathogenesis of abnormalities in peripheral nerves in patients with hypothyroidism include endoneurial accumulation of aminoglycane, as well as mucinous deposits in soft tissue surroundings of the peripheral nerves. These contribute to increased pressure on the median nerve at the wrist level $(1,9)$. Hypothyroidism produces alterations of fluid balance and peripheral tissue edema, which may lead to CTS development $(1,12)$. However, there are no data how autoimmunity plays a role in CTS pathogenesis. Consequently, treatment of hypothyroidism may help to minimize or cure CTS complaints $(1,12,13,14)$. Diagnosis is usually based on clinical symptoms and physical examination maneuvers, and is supported by nerve conduction studies (NCSs). In the literature review by the American Association of Neuromuscular and Electrodiagnostic Medicine (AANEM) on the diagnosis of CTS, the sensitivity and specificity of NCS was 85 and 95\% respectively (15). Ultrasonography (US) has been used as an alternative to NCS in the diagnosis of CTS (16). Despite some limitations, US of median nerve has not only been considered for the diagnosis of CTS, but also for the identification of the severity of median nerve damage. In comparison with NCS, US measurements from different sites were most consistent, and best sensitivity and specificity was reported for measures of cross-sectional area (CSA) of median nerve at the level of os pisiforme (17). Various studies have demonstrated that CSA of the median nerve at the carpal tunnel inlet and carpal tunnel outlet is significantly greater in CTS patients than in the normal population $(15,18,19,20)$. The CSA of the median nerve at the carpal tunnel inlet (at the level of the pisiform) is the most sensitive and specific US finding in patients with CTS. The cut-off value of CSA at the tunnel inlet in patients with CTS ranges from 6.5 to $15 \mathrm{~mm}^{2}(19,20,21,22)$. This difference in the cut-off point values of the tunnel inlet is due to the heterogeneity of study designs, different sample sizes, patient's characteristics and operator experience (21). Median nerve enlargement $\left(C S A \geq 10 \mathrm{~mm}^{2}\right.$ at the level of the pisiform bone or tunnel inlet) is the most commonly used parameter to diagnose CTS on US, and sensitivity has been reported to be as high as $97.9 \%$ using this parameter (16). To our knowledge, there have been no studies performed investigating autoimmune hypothyroidism and CSA using US. This study aims to provide novel information concerning US evaluation of CTS.
The primary objective of the study was to assess median nerve entrapment in patients with newly diagnosed and untreated autoimmune hypothyroidism in comparison with healthy adults. Furthermore, we assessed changes in the CSA by US before and following thyroxine treatment.

\section{Patients and methods}

\section{Patients}

We performed a cross-sectional, prospective follow-up study on 30 consecutive patients newly diagnosed with primary autoimmune hypothyroidism at the 5th Department of Internal Medicine, Medical Faculty of Comenius University, University Hospital Bratislava. The control group comprised age-, sex-, and BMI (Body mass index)-matched healthy controls. The control subjects had no history of any thyroid disorders and had a normal serum TSH and free $\mathrm{T}_{4}$. The study protocol was approved by the ethics committee of the University Hospital Bratislava, and informed consent was obtained from all patients and controls according to the World Medical Association Declaration of Helsinki, revised in 2000, Edinburgh.

Inclusion criteria were as follows: newly diagnosed and untreated autoimmune hypothyroidism defined by thyroid-stimulating hormone (TSH) levels greater than $4.78 \mathrm{mIU} / \mathrm{L}$ and aTG plasma levels greater than $60 \mathrm{kIU} / \mathrm{L}$ and/or aTPO plasma levels greater than $60 \mathrm{kIU} / \mathrm{L}$. No prior history or presence of any thyroid disorder or other conditions that may cause compressive neuropathy (e.g. diabetes mellitus, acromegaly, paraneoplastic, inflammation, alcoholism, liver and kidney disease, as well as mechanical overload) documented in patients cohort.

Laboratory investigations including complete blood count, serum glucose, creatinine, electrolytes, liver and kidney enzymes, vitamin B12 and folic acid were performed for all patients at baseline to eliminate other possible causes of neuropathy.

All patients received thyroxine treatment (mean: $83.5 \mu \mathrm{g}$, range: $25-125 \mu \mathrm{g}$ ) for hypothyroidism, and TSH and $\mathrm{fT}_{4}$ levels were monitored for 3 months following baseline. Patients in control group underwent US evaluation at baseline and following 3 months.

\section{Outcome measures}

Thyroid function was assessed by measuring the concentration of TSH using a solid-phase, monoclonal 
antibody-labeled chemiluminescent immunoassay (CLIA) (ADVIA Centaur and ADVIA Centaur XP; Tarrytown, NY, interassay coefficient of variability (CV), 3.55-5.51\%); $\mathrm{fT}_{4}$ using a solid-phase, polyclonal antibody-labeled CLIA (ADVIA Centaur and ADVIA Centaur XP; Tarrytown, NY, USA, interassay CV, 3.44-4.16\%); thyroid peroxidase antibodies (TPO-Ab) using an electrochemiluminescence immunoassay (ECLIA) (Elecsys a cobas e analyzers, Basel, Swiss; interassay CV, 5.6-8.9\%); and thyroglobulin antibodies (TG-Ab) using ECLIA (Elecsys and cobas e analyzers, Basel, Swiss; interassay CV, 5.8-6.0\%).

\section{Ultrasound}

All patients underwent high-resolution real-time US of the carpal tunnel before and after thyroxine therapy. Ultrasound measurements were performed by a single operator using a $12 \mathrm{MHz}$ linear array transducer (GE LOGIQ e portable ultrasound machine, Willowick, $\mathrm{OH}$, USA). The US examination was performed with the patient seated in front of the sonographer, with the forearm resting on the table and the palm facing up in the neutral position. We performed transverse imaging of the median nerve for the area of the carpal tunnel inlet, which is defined as the proximal margin of the flexor retinaculum between the scaphoid tubercle and the pisiform bone. A longitudinal view was used to confirm the correct identification of the median nerve. The median nerve was observed on the screen as an oval or ellipsoid hypoechoic reticular area with a hyperechoic rim beneath the flexor retinaculum (19). The median nerve CSA was measured by the direct method (tracing method). Each measurement was performed five times. To prevent measurement error, the highest and lowest values were eliminated, and the remaining three measurements were averaged. This is the most extensive methodology for measuring the median nerve encountered among the studies (23).

\section{Statistics}

Statistical analysis was performed using the statistics software SPSS version 19 (IBM SPSS Statistics, IBM). Categorical data were expressed as the mean \pm standard error of the mean (s.E.M.). Unpaired $t$-tests were performed for comparisons between the hypothyroidism subject and his/her control subject who was matched for age, sex and BMI. Paired $t$-test was used to compare baseline and post-treatment results in the hypothyroid group. Pearson correlation coefficients were calculated to analyze the correlation between TSH, thyroid autoantibodies and CSA of the nervus medianus.

\section{Results}

\section{Study group}

Thirty patients were included in the study at baseline and comprised 22 women and 8 men. In the control group, 22 women and 8 men were included. The mean age of patients was 49.2 years, and in the control group, 52.6 years. No significant difference in sex, BMI and age was found between both the patient and control groups. Before thyroxine treatment, a significantly greater median nerve CSA of patients in comparison with controls $\left(9.8 \pm 0.7 \mathrm{~mm}^{2}\right.$ vs $\left.7.2 \pm 1.1 \mathrm{~mm}^{2} ; P<0.001\right)$ was observed (Table 1). In patients cohort, CSA $>11 \mathrm{~mm}^{2}$ in four patients was recorded. Significant differences were found between TSH levels at baseline $(23.9 \pm 6.0 \mathrm{mIU} / \mathrm{L})$ and after 3 months $(2.7 \pm 0.2 \mathrm{mIU} / \mathrm{L} ; P<0.001)$. In the cohort of patients, a number of 16 had TSH $>10 \mathrm{mIU} / \mathrm{L}$ and 14 patients had normal levels of $\mathrm{fT}_{4}$. In those 14 subjects, median nerve CSA was greater in comparison with controls $\left(9.8 \pm 0.4 \mathrm{~mm}^{2}\right.$ vs $\left.7.2 \pm 1.1 \mathrm{~mm}^{2} ; P<0.001\right)$. In entire study group after thyroxine treatment, median nerve CSA significantly decreased $\left(9.8 \pm 0.7 \mathrm{~mm}^{2}\right.$ vs $\left.8.4 \pm 0.3 \mathrm{~mm}^{2} ; P=0.033\right)$. After treatment, no difference in BMI was observed $\left(24.5 \pm 0.8 \mathrm{~kg} / \mathrm{m}^{2}\right.$ vs $\left.24.4 \pm 0.7 \mathrm{~kg} / \mathrm{m}^{2}\right)$ (Table 2). None of the patients had BMI $>30 \mathrm{~kg} / \mathrm{m}^{2}$ at baseline and after treatment.

There was a relationship between hypothyroidism and median nerve CSA. At baseline, a positive correlation was observed between TSH and CSA $(R=0.155, P=0.05)$ (Fig. 1), and a negative correlation was found between $\mathrm{fT}_{4}$ and CSA ( $R=-0.149, P=0.001)$ (Fig. 2$)$. No correlation was

Table 1 Baseline characteristics of patients and control groups. Data is presented as means or mean \pm S.E.M.

\begin{tabular}{|c|c|c|c|}
\hline Variable & $\begin{array}{c}\text { Patients } \\
(n=30)\end{array}$ & $\begin{array}{c}\text { Control } \\
(n=30)\end{array}$ & $P$-value \\
\hline $\operatorname{Sex}(M / F)$ & $8 / 22$ & $8 / 22$ & $>0.05$ \\
\hline Age (year) & $49.2 \pm 2.3$ & $52.6 \pm 2.9$ & \\
\hline $\mathrm{M} / \mathrm{F}$ (mean) & $55.9 / 46.7$ & $59.4 / 49.4$ & $>0.05$ \\
\hline BMI $\left(\mathrm{kg} / \mathrm{m}^{2}\right)$ & $24.5 \pm 0.7$ & $24.5 \pm 0.5$ & $>0,05$ \\
\hline M/F (mean) & $27.1 / 23.5$ & $26.4 / 23.6$ & $>0.05$ \\
\hline TSH (mIU/L) & $23.8 \pm 6.0$ & $2.3 \pm 0.2$ & \\
\hline M/F (mean) & $33.9 / 20.2$ & $2.3 / 2.3$ & $<0.001$ \\
\hline $\mathrm{fT}_{4}(\mathrm{pmol} / \mathrm{L})$ & $11.0 \pm 0.6$ & $16.5 \pm 0.4$ & $<0001$ \\
\hline M/F (mean) & $10.6 / 11.1$ & $16.5 / 16.4$ & $<0.001$ \\
\hline $\mathrm{CSA}\left(\mathrm{mm}^{2}\right)$ & $9.8 \pm 0.7$ & $7.2 \pm 1.1$ & \\
\hline M/F (mean) & $10.3 / 9.7$ & $8 / 7$ & $<0.001$ \\
\hline
\end{tabular}


Table 2 Body-mass index, levels of thyroid-stimulating hormone and free thyroxine, and cross-sectional area before and after treatment with thyroxine. Data are presented as mean \pm S.E.M.

\begin{tabular}{l}
\hline Variable \\
\hline BMI $\left(\mathrm{kg} / \mathrm{m}^{2}\right)$ \\
TSH $(\mathrm{mlU} / \mathrm{L})$ \\
$\mathrm{fT}_{4}(\mathrm{pmol} / \mathrm{L})$ \\
$\mathrm{CSA}\left(\mathrm{mm}^{2}\right)$ \\
\hline
\end{tabular}

$\begin{array}{r}\hline \text { Baseline } \\ \hline 24.5 \pm 0.8 \\ 23.9 \pm 6.0 \\ 11.0 \pm 0.6 \\ 9.8 \pm 0.7 \\ \hline\end{array}$

\begin{tabular}{|c|c|}
\hline Month 3 & P-value \\
\hline $24.4 \pm 0.7$ & $>0.05$ \\
\hline $2.7 \pm 0.2$ & $<0.001$ \\
\hline $15.5 \pm 0.4$ & $<0.001$ \\
\hline $8.4 \pm 0.3$ & 0.003 \\
\hline
\end{tabular}

observed between TSH, $\mathrm{fT}_{4}$ and CSA after normalization of thyroid function after 3 months. This result was expected as a common physiological finding.

In four hypothyroid patients, clinical signs (e.g. distal paresthesias with weakness, tingling and pain in the distribution of the median nerve) of CTS were reported. Two of them had a baseline CSA measurement greater than $11 \mathrm{~mm}^{2}$, which is considered pathognomonic for CTS. Following 3 months of appropriate hormone replacement, CSA values were normalized in these patients, and their symptoms resolved.

\section{Discussion}

Previous studies of ultrasound assessment of median nerve confirmed greater median nerve CSA in patients with thyroid dysfunction in comparison with healthy

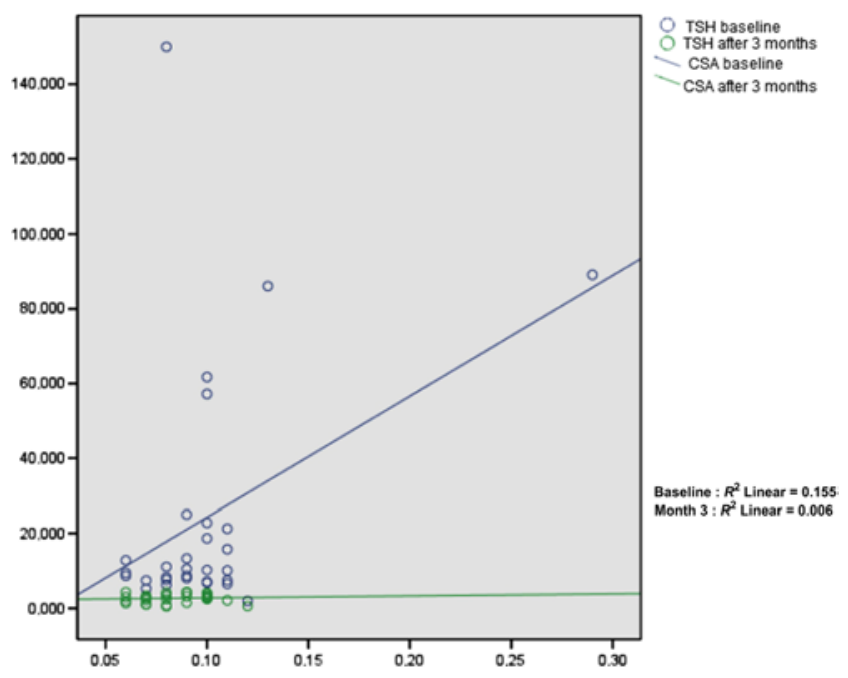

Figure 1

Trend in CSA - TSH relationship. At baseline, a positive correlation was observed between TSH and CSA $(R=0.155$, $P=0.05)$. A full colour version of this figure is available at http://dx.doi.org/10.1530/EJE-16-0397.

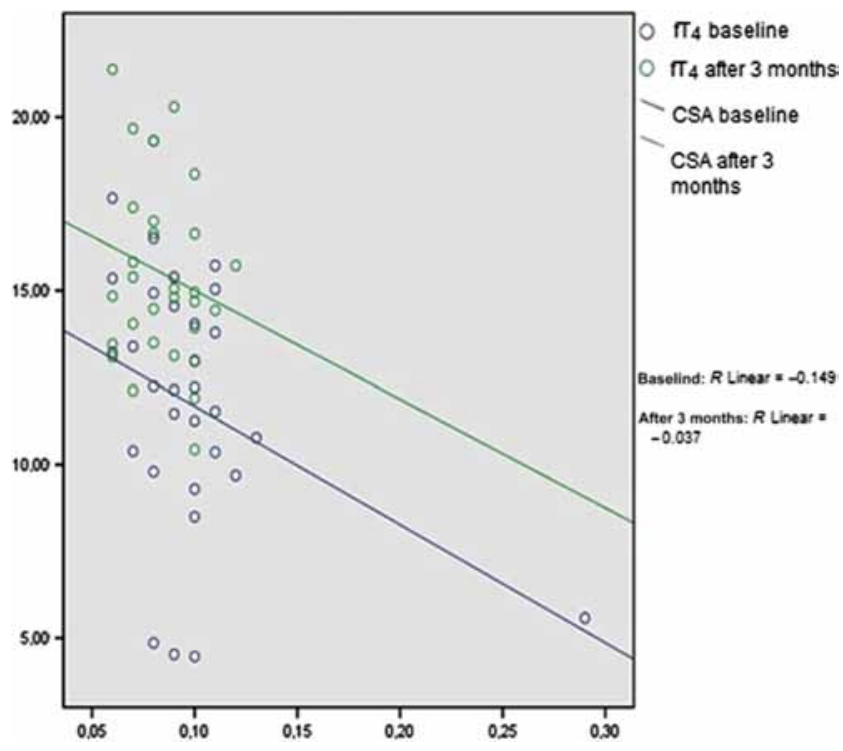

\section{Figure 2}

Trend in CSA $-\mathrm{fT}_{4}$ relationship. At baseline, a negative correlation was found between $\mathrm{fT}_{4}$ and CSA $(R=-0.149$, $P=0.001)$. A full colour version of this figure is available at http://dx.doi.org/10.1530/EJE-16-0397.

controls. However, no previous study on thyroid hormone replacement-mediated effect on CSA was reported.

In this study, we performed ultrasound measurement of the median nerve CSA in patients which are due to thyroid dysfunction at higher risk of developing CTS, followed by US measurements after 3-month thyroxine treatment. The strengths of the study are prospective study design of consecutively included hypothyroid patients with carefully matched control group and using of high-tech US performed by a single examiner. We are aware of some limitations e.g. smaller sample size with low number of patients with clinical signs of CTS and presence of subclinical hypothyroidism in some patients.

Recent studies have shown that US is effective in confirming the diagnosis of CTS $(24,25,26)$. Ultrasound has several advantages compared with NCS. First, other lesions that display symptoms similar to CTS may be excluded from examination, and these include tenosynovitis, mass lesions and anatomic defects. Furthermore, US is cheaper, widely available, involves a short examination time and it is more sensitive and less invasive than NCS. The use of US can be recommended as the first-line method in the diagnosis of CTS (24).

In this study, all patients with hypothyroidism were found to have significantly higher TSH levels and lower $\mathrm{fT}_{4}$ levels compared with the control group. At baseline, 
US measurement of the CSA at the level of the pisiform bone demonstrated a mean value of $9.8 \mathrm{~mm}^{2}$ in the patient group compared with $7.2 \mathrm{~mm}^{2}$ in the control group, which is comparable to results from previous studies $(15,19,25,26,27)$. In the study group, 14 patients had $\mathrm{fT}_{4}$ levels in normal range. Accumulation of mucopolysaccharides in the tendon sheaths and synovial membranes may cause CTS by loading pressure on the median nerve. It is obvious that this condition is more common in long-standing disease (28). Some studies have shown that the neuromuscular signs of hypothyroidism may be present in the subclinical forms as well $(29,30$, 31). Despite this fact, a similar study by Jalilzadeh et al. indicated that there were no neuropathic or myopathic changes in patients with subclinical hypothyroidism, besides CTS (32). Study by Shiri using neuromuscular studies showed that symptoms of CTS are also seen in patients with hypothyroidism when they are euthyroid or they are on thyroid replacement therapy, but CTS was not a consequence of subclinical hypothyroidism (33). However, this study was aimed to detect the median nerve thickening measured by ultrasound regardless of clinical manifestation of thyroid disease or CTS. When statistical analysis was performed in subset of patients with normal $\mathrm{fT}_{4}$ levels and increased $\mathrm{TSH}$, the results for median nerve CSA still remain significantly elevated in comparison with healthy controls.

Changes in the BMI may potentially affect the integrity of the nerve. Werner et al. demonstrated that obese individuals (BMI>29) are 2.5 times more likely to complain of CTS than slender individuals $(\mathrm{BMI}<20)(34)$. The correlation between the CSA of the median nerve with BMI may exist. In this study, no significant difference in BMI was observed between patients and the control group; hence, our results do not appear to be influenced by this factor.

In our patient cohort with hypothyroidism, the CSA of the median nerve was greater in men $\left(10.3 \pm 0.4 \mathrm{~mm}^{2}\right)$ compared with women $\left(9.7 \pm 0.5 \mathrm{~mm}^{2}\right)$. The CSA of the median nerve was greater in men compared with women in control group as well. This result is similar to the study of 49 patients, who reported the CSA of the median nerve proximal to the carpal tunnel to be $2.2 \mathrm{~mm}^{2}$ higher in men than in women, although these patients did not have hypothyroidism (35).

To the best of our knowledge, there is no study comparing the effect of thyroxine treatment on the CSA in patients with primary hypothyroidism. We have performed a prospective follow-up study of patients treated with thyroxine over a 3-month period. The results confirmed a significant decrease in TSH levels and CSA values after thyroxine treatment, with no change in BMI. At baseline, a significant correlation was observed between $\mathrm{TSH}, \mathrm{fT}_{4}$ levels and CSA values, although this relationship was not observed following normalization of TSH and $\mathrm{fT}_{4}$. This relationship confirmed that the CSA values in patients with overt hypothyroidism are directly influenced by levels of thyroid hormones.

Furthermore, at baseline, four patients were reported to have distal paresthesia in the distribution of median nerve, with weakness, tingling and pain. Pathologically increased median nerve CSA values (greater than $11 \mathrm{~mm}^{2}$ ) were documented in two of these patients. At 3 months, CSA values and clinical symptoms of CTS become normal in these four patients. This additional evaluation provides the opportunity to evaluate the efficacy of replacement therapy on the enlargement of the CSA of the median nerve, and aids in the prevention of unnecessary surgery in primary hypothyroidism patients with clinical signs of CTS. Follow-up of these patients demonstrated that the abnormalities related to entrapment neuropathy in hypothyroid patients can be reversed with adequate treatment.

This study demonstrated the influence of autoimmune hypothyroidism on structural alterations of the nerve, represented by US determinants of median nerve CSA, and thus evaluated the adverse effect of hypothyroidism on the median nerve. This study provides coherent data on the effect of primary hypothyroidism on the enlargement of the median nerve CSA. The data were evaluated with respect to median nerve CSA measurements and compared with BMI-, age- and sexmatched controls. Patients with untreated primary hypothyroidism were found to have a significantly greater CSA compared with healthy controls. In addition, the presence of entrapment neuropathy in hypothyroidism decreased after replacement treatment in newly diagnosed patients. The study provided a support to a well-known clinical observation that adequate treatment helps to prevent surgical decompression in CTS induced by hypothyroidism. Early treatment of hypothyroidism may impede the progression of neuromuscular complications or minimize their occurrence. Autoimmunity may potentially play an important role in the pathogenesis of nerve thickening associated with hypothyroidism; however, this has not yet been investigated. Ultrasound is a sensitive, noninvasive method for the diagnosis of CTS associated with hypothyroidism providing a metric which improves with substitution treatment of hypothyroidism, thus supporting the pathophysiology and clinical findings. 
Declaration of interest

The authors declare that there is no conflict of interest that could be perceived as prejudicing the impartiality of the research reported.

\section{Funding}

This research did not receive any specific grant from any funding agency in the public, commercial or not-for-profit sector.

Acknowledgments

The authors would like to acknowledge Dr Michal Chovanec for critical input during manuscript writing.

\section{References}

1 Kasem AA, Fathy SM, Shahin DA \& Fikry AA. Carpal tunnel syndrome in hypothyroid patients: the effect of hormone replacement therapy. American Journal of Internal Medicine 20142 54-58. (doi:10.11648/j. ajim.20140203.14)

2 Lazúrová I, Benhatchi K, Rovenský J, Kozáková D, Wagnerová H, Tajtáková M, Shoenfeld Y \& Macejova Z. Autoimmune thyroid disease and autoimmune rheumatic disorders: a two-sided analysis. Annals of the New York Academy of Sciences 20091173 211-216. (doi:10.1111/ j.1749-6632.2009.04809.x)

3 Appenzeller S, Shoenfeld Y \& de Carvalho JF. Neurologic manifestation of autoimmune diseases. Autoimmune Diseases 2012 2012 683212. (doi:10.1155/2012/683212)

4 Yeasmin S, Begum N, Begum S \& Rahman SMH. Relationships of nerve conduction parameters with the thyroid hormones in hypothyroid patients. Journal of Bangladesh Society of Physiologist 2013 8 1-5. (doi:10.3329/jbsp.v8i1.16638)

5 Eslamian F, Bahrami A, Aghamohammadzadeh N, Niafar M, Salekzamani Y \& Behkamrad K. Electrophysiologic changes in patients with untreated primary hypothyroidism. Journal of Clinical Neurophysiology 201128 323-328. (doi:10.1097/ WNP.0b013e31821c30d9)

6 Dijk MAJ, Reitsma JB, Fischer JC \& Sanders GTB. Indications for requesting laboratory tests for concurrent diseases in patients with carpal tunnel syndrome: a systematic review. Clinical Chemistry 2003 49 1437-1444. (doi:10.1373/49.9.1437)

7 El-Salem K \& Ammari F. Neurophysiological changes in neurologically asymptomatic hypothyroid patients: a prospective cohort study. Journal of Clinical Neurophysiology 200623 568-572. (doi:10.1097/01. wnp.0000231273.22681.0e)

8 Duyff RF, Van den Bosch J, Laman DM, van Loon BJB \& Linssen WHJP. Neuromuscular findings in thyroid dysfunction: a prospective clinical and electrodiagnostic study. Journal of Neurology, Neurosurgery and Psychiatry 200068 750-755. (doi:10.1136/ jnnp.68.6.750)

9 Ajeena IM. Prevalence of neuromuscular abnormalities in newly diagnosed patients with thyroid dysfunction. American Journal of Research Communication 20131 79-88.

10 Aroori S \& Spence RAJ. Carpal tunnel syndrome. Ulster Medical Journal 200877 6-17.

11 American Academy of Orthopedic Surgeons. Clinical practice guidelines on diagnosis of carpal tunnel syndrome. In American Academy of Orthopedic Surgeons, edn 1, p 3. Rosemont, IL, USA, 2007.

12 Kececi H \& Degirme Y. Hormone replacement therapy in hypothyroidism and nerve conduction study. Journal of Clinical Neurophysiology 200636 79-83. (doi:10.1016/j.neucli.2006.04.001)

13 Palumbo CF, Szabo RM \& Olmsted SL. The effects of hypothyroidism and thyroid replacement on the development of carpal tunnel syndrome. Journal of Hand Surgery 200025 734-739. (doi:10.1053/ jhsu.2000.8642)

14 Fathy SM, Shahin D \& Kasem AA. The effect of hormone replacement therapy in hypothyroid patients with carpal tunnel syndrome. Anveshana Ayurveda Medical Journal 20108 156-170.

15 Chan KY, George J, Goh KJ \& Ahmad TS. Ultrasonography in the evaluation of carpal tunnel syndrome: diagnostic criteria and comparison with nerve conduction studies. Neurology Asia 201116 57-64.

16 McDonagh C, Alexander M \& Kane D. The role of ultrasound in the diagnosis and management of carpal tunnel syndrome: a new paradigm. Rheumatology 201554 9-19. (doi:10.1093/rheumatology/ keu275)

17 El Miedany Y, El Gaafary M, Youssef S, Ahmed I \& Nasr A. Ultrasound assessment of the median nerve: a biomarker that can help in setting a treat to target approach tailored for carpal tunnel syndrome patients. SpringerPlus 20154 1-10. (doi:10.1186/2193-1801-4-1)

18 Kwon BCH, Jung K \& Baek GH. Comparison of sonography and electrodiagnostic testing in the diagnosis of carpal tunnel syndrome. Journal of Hand Surgery 2008 33A 65-71. (doi:10.1016/j. jhsa.2007.10.014)

19 Wang LY, Leong CHP, Huang YCH, Hung JW, Cheung SM \& Pong YP. Best diagnostic criterion in high-resolution ultrasonography for carpal tunnel syndrome. Chang Gung Medical Journal 200831 469-476.

20 Wong SM, Griffith JF, Hui ACF, Tang A \& Wong KS. Discriminatory sonographic criteria for the diagnosis of carpal tunnel syndrome. Arthritis and Rheumatology 200246 1914-1921. (doi:10.1002/art.10385)

21 Sarraf P, Malek M, Ghajarzadeh M, Miri S, Parhizgar E \& Razavi SZE. The best cutoff point for median nerve cross sectional area at the level of carpal tunnel inlet. Acta Medica Iranica 201452 613-618.

22 Fowler JR, Gaughan JP \& Ilyas AM. The sensitivity and specificity of ultrasound for the diagnosis of carpal tunnel syndrome: a meta-analysis. Clinical Orthopaedics and Related Research 2011469 1089-1094. (doi:10.1007/s11999-010-1637-5)

23 Roll SC, Case-Smith J \& Evans KD. Diagnostic accuracy of ultrasonography vs electromyography in carpal tunnel syndrome: systematic review of literature. Ultrasound in Meicine \& Biology 201137 1539-1553. (doi:10.1016/j.ultrasmedbio.2011.06.011)

24 Ghasemi-rad M, Nosair E, Vegh A, Mohammadi A, Akkad A, Lesha E, Mohammadi MH, Sayed D, Davarian A, Maleki-Miyandoab T et al. A handy review of carpal tunnel syndrome: from anatomy to diagnosis and treatment. World Journal of Radiology $20146284-300$. (doi:10.4329/wjr.v6.i6.284)

25 Wong SM, Griffith JF, Hui ACF, Kai Lo S, Fu M \& Wong KS. Carpal tunnel syndrome: diagnostic usefulness of sonography. Radiology 2004232 93-99. (doi:10.1148/radiol.2321030071)

26 El Miedany YE, Aty SA \& Ashour S. Ultrasonography versus nerve conduction study in patients with carpal tunnel syndrome: substantive or complementary tests? Rheumatology $2004 \mathbf{4 3}$ 887-895 (doi:10.1093/rheumatology/keh190)

27 Swen WA, Jacobs JW, Bussemaker FE, de Waard JW \& Bijlsma JW. Carpal tunnel sonography by the rheumatologist versus nerve conduction study by the neurologist. Journal of Rheumatology $2001 \mathbf{2 8}$ 62-69.

28 Gedizlioglu M, Ozerkan E, Kulan CA \& Karasa B. Bilateral carpal tunnel syndrome and thyroid functions. Nörolojik Bilimler Dergisi 2001 18 1-8.

29 Akarsu EO, Acar H, Ozer F, Günaydın S, Akarsu Ö, Özcan TA, Özben S, Mutlu A, Bedir M, Gül GÇ et al. Electromyographic findings in overt hypothyroidism and subclinical hypothyroidism. Turkish Journal of Neurology 201319 128-133. (doi:10.4274/tnd.05658)

30 Monzani F, Caraccio N, Del Guerra P, Casolaro A \& Ferrannini E. Neuromuscular symptoms and dysfunction in subclinical hypothyroid patients: beneficial effect of L-T4 replacement therapy. Clinical Endocrinology 19992 237-242. (doi:10.1046/j.13652265.1999.00790.x) 
31 Raza SA \& Mahmood N. Subclinical hypothyroidism: controversies to consensus. Indian Journal of Endocrinology and Metabolism $2013 \mathbf{1 7}$ 636-642. (doi:10.4103/2230-8210.123555)

32 Jalilzadeh SH, Bahrami A \& Eftekharosadat B. Elektrophysiologic changes in patients with subclinical hypothyroidism (in Persian). Medical Journal of Tabriz University of Medical Sciences 200729 37-41.

33 Shiri R. Hypothyroidism and carpal tunnel syndrome: a metaanalysis. Muscle and Nerve 20146 1-5.
34 Werner RA, Albers JW, Franzblau A \& Armstrong TJ. The relationship between body mass index and the diagnosis of carpal tunnel syndrome. Muscle and Nerve 199417 632-636. (doi:10.1002/mus. v17:6)

35 Klauser AS, Halpern EJ, Faschingbauer R, Guerra F, Martinoli C, Gabl MF, Arora R, Bauer T, Sojer M, Loscher WN et al. Bifid median nerve in carpal tunnel syndrome: assessment with US cross-sectional area measurement. Radiology 2011259 123-129.

Received 8 May 2016

Revised version received 22 June 2016

Accepted 14 July 2016 